\author{
ANNALES \\ POLONICI MATHEMATICI \\ XXIX (1974)
}

\title{
Tadeusz Ważewski (1896-1972)
}

\author{
par C. Olech, J. Szarski et Z. SzmydT
}

Le 5 septembre 1972 est décédé à Zaryte Tadeusz Ważewski, illustre géomètre, professeur à l'Université Jagellone de Cracovie, un des fondateurs de l'Institut Mathématique de l'Académie Polonaise des Sciences, membre de l'Académie Polonaise des Sciences, fondateur de l'école des équations différentielles de Cracovie.

Tadeusz Ważewski est né Je 24 septembre 1896 à Wygnanka. Ayant terminé le lycée à Tarnów en 1914, il a commencé ses études à l'Université Jagellone. Il étudiait d'abord la physique et ensuite il a entrepris les études mathématiques sous la direction de Stanisław Zaremba. Son intérêt fut attiré par la théorie des ensembles et la topologie qui se développaient à cette époque, sans être cultivées à Cracovie. En 1920 fut publié son travail sur un continu singulier [1]. Dans la période 1921-1923 Ważewski continuait ses études à Paris. En 1924 il a obtenu le grade du docteur ès sciences mathématiques à l'Université de Paris, en soutenant la thèse sur les soit-disants dendrites [3], e'est-à-dire les continus localement connexes ne contenant aucune courbe simple, fermée. Les membres de la commission d'examen étaient: E. Borel, A. Denjoy et P. Montel.

En 1927 Ważewski a passé son examen de professorat à l'Université Jagellone, en présentant la thèse sur les continus rectifiables [4]. Ses travaux suivants concernaient déjà l'analyse mathématique. Parmi ses premiers travaux de ce domaine il y a deux qui méritent particulièrement d'être mentionnés. Dans une [8] de ces notes Ważewski a introduit la notion des jacobiens asymptotiques et dans l'autre [12] il s'en est servi pour démontrer le théorème sur le changement des variables dans l'intégrale multiple sans l'hypothèse que la transformation soit inversible.

En 1933 Ważewski fut nommé professeur extraordinaire à l'Université Jagellone. Dans cette période il a commencé à s'intéresser aux équations différentielles, domaine de mathématique dans lequel devait se révéler la grandeur de ses découvertes scientifiques. Ce changement du domaine des recherches s'était réalisé partiellement sous l'influence de Stanisław Zaremba, mais e'était surtout l'effet de l'intérêt que Ważewski trouvait aux phénomènes de la nature et du besoin qu'il sentait pour 
mettre ces phénomènes en forme mathématique. Ces domaines des mathématiques qui ont des rapports avec d'autres branches des mathématiques et avec d'autres sciences, étaient considérés par Ważewski comme les plus importants. Dans un de ses discours, il a dit: „La nature a le caractère mathématique. Sans mathématiques, il serait impossible de donner aux lois de la nature une forme quantitative. C'est justement cette forme quantitative qui seule peut rendre services à la technique".

Ważewski était un chercheur extrêmement sagace. Il savait pénétrer profondement la nature du problème, mettre en évidence son sens essentiel et décourrir des analogies entre des problèmes en apparence distants. Grâce à ces qualités Ważewski est devenu spécialiste éminent dans le domaine des équations différentielles, domaine qu'il enrichissait jusqu'au terme de ses jours des idées d'une originalité extraordinaire et dans lequel il entamait des nouvelles directions des recherches.

Parmi les travaux portant sur les équations différentielles et publiés avant 1939 il y a trois particulièrement intéressants, dans lesquels Ważewski appliquait, pour la première fois, d'une façon systématique la théorie des inégalités différentielles ordinaires à celle des équations aux dérivées partielles du premier orde. La première note [17] concerne un critère général, du type de Kamke, de l'unicité de la solution du problème de Cauchy pour les équations non linéaires aux dérivées partielles du premier ordre; la seconde note [30] contient l'appréciation effective du domaine de l'existence d'une telle solution sous les faibles hypothèses de régularité. La troisième note [33], concernant l'existence de la solution du problème de Cauchy pour un système diagonal d'équations aux dérivées partielles du premier ordre, contient le premier résultat sur ce sujet dans la littérature mathématique. Avant 1939 fut aussi publié le travail [16] donnant un résultat négatif concernant l'existence globale de l'intégrale première pour l'équation différentielle ordinaire. Ważewski a construit notamment l'équation différentielle $y^{\prime}=f(x, y)$, dont le second membre est de la classe $C^{\infty}$ dans un domaine $\Omega$, pour laquelle chaque intégrale première de classe $C^{1}$ dans $\Omega$ est forcément constante. Après la guerre ce résultat fut le point de départ pour des nombreuses publications des élèves de Ważewski.

En octobre 1939 Tadeusz Ważewski se trouva dans le groupe de professeurs des écoles supérieures de Cracovie qui ont été arrêtés par les fascistes allemands et ensuite transportés au camp de concentration à Oranienburg et où un grand nombre en a perdu la vie. Voici ce qu'a dit à propos de Ważewski Kazimierz Stołyhwo, antropologue polonais de grande renommée, qui a été aussi prisonnier au camp à Oranienburg: „J'aime beaucoup la ville de Cracovie, parceque Tadeusz Ważewski y habite, et je ne regrette pas d'avoir été prisonnier à Oranienburg, parce que là-bas j'ai fait connaissance de Ważewski". Il faut mentionner que par 
suite des interventions de maints savants Stołyhwo aurait pu être mis en liberté beaucoup plus tôt, mais il a refusé en disant qu'il ne pouvait pas quitter le camp tant que ses collègues y restaient.

Après son retour à Cracovie Ważewski enseignait à l'université clandestine en continuant intensivement ses recherches scientifiques en dépit des dures conditions de l'occupation fasciste. A cette période appartiennent les travaux concernant l'appréciation du domaine d'existence des fonctions implicites [40] ainsi que le mémoire fondamental sur les inégalités différentielles ordinaires [57]. Ce dernier travail a déterminé une des directions des recherches menées après la guerre dans le centre mathématique de Cracovie.

En 1945 Tadeusz Ważewski fut nommé professeur ordinaire à l'Université Jagellone. Pendant les premières années après la guerre son attention a été attirée par les problèmes du parcours qualitatif des solutions des équations différentielles ordinaires. Pendant cette période Ważewski a créé une nouvelle méthode topologique pour examiner l'allure qualitative des solutions [48]. Cette méthode faisant intervenir la notion de retracte introduite par Karol Borsuk, s'appuie sur une idée simple mais profonde et s'est montrée très féconde dans les applications. Nous allons l'illustrer sur un exemple élémentaire.

Considérons un système de deux équations différentielles

$$
d x / d t=f(t, x),
$$

où $x=\left(x_{1}, x_{2}\right), f=\left(f_{1}, f_{2}\right)$, dont les solutions sont univoquement déterminées par les conditions initiales dans un ensemble ouvert contenant le parallélépipède

$$
-\infty<t<\infty, \quad\left|x_{1}\right| \leqslant 1, \quad\left|x_{2}\right| \leqslant 1 .
$$

Supposons que $x_{1} f_{1}>0$ pour $\left|x_{1}\right|=1$ tandis que $x_{2} f_{2}<0$ pour $\left|x_{2}\right|=1$. Ces hypothèses veulent dire que par les faces opposées du parallélépipède (2): $A$ sur laquelle $x_{1}=1$ et $B$ sur laquelle $x_{1}=-1$, les intégrales du système (1) sortent de l'ensemble (2), tandis que par les deux autres faces elles $y$ entrent. Le théorème de Ważewski dit que dans ces conditions sur chaque segment joignant les faces $A$ et $B$ il existe un point $p$ tel que l'intégrale issue de $p$ reste dans l'ensemble (2) pour $t \rightarrow+\infty$. Les raisons, pour lesquelles il en est ainsi, sont bien simples. Dans l'exemple considéré les intégrales du système (1) issues de l'intérieur de l'ensemble (2) ne peuvent en sortir que par les faces $A$ et $B$. Choisissons sur ces faces deux point $a \in A$ et $b \in B$. Si l'intégrale issue d'un point $p$ de l'ensemble (2) en sort pour la première fois par la face $A$, alors faisons correspondre à $p$ le point $a$; si elle en sort pour la première fois par la face $B$, alors faisons correspondre à $p$ le point $b$. De cette façon nous avons défini dans un sous-ensemble $E$ de (2) une application continue. L'intersection de $E$ avec 
le segment joignant les points $a$ et $b$ est différente de ce segment; en effet dans le cas contraire notre application transformerait d'une façon continue le segment sur ses extrémités, ce qui est impossible. Par conséquent, il existe un point $p$ de ce segment tel que l'intégrale issue de $p$ ne quitte pas l'ensemble (2) pour $t \rightarrow+\infty$.

La méthode de retracte, due à T. Ważewski, s'est montrée très efficace dans l'examen des problèmes asymptotiques de los théorie des équations différentielles ordinaires. Elle devint le point de départ pour les investigations de l'auteur lui-même, de ses élèves et des spécialistes à l'étranger. Jusqu'aujourdhui de nouvelles généralisations de la méthode trouvent des applications dans maints nouveaux problèmes, aussi dans la théorie des équations aux dérivées partielles. La méthode topologique de Ważewski est entrée dans les monographies et les manuels de la littérature mathématique mondiale. Sur l'invitation du Comité d'Organisation Tadeusz Ważewski a donné au Congrès International des Mathématiciens, tenn à Amsterdam en 1954, une conférence sur sa méthode [80].

La méthode de retracte compte parmi les plus grands succès de la mathématique polonaise après la seconde guerre mondiale. D'après l'opinion de Solomon Lefschetz, illustre mathématicien américain, exprimée en 1961, la méthode de retracte de T. Ważewski est une des plus grandes découvertes dans la théorie des équations différentielles ordinaires après 1945 .

C'était un trait caractéristique de l'activité scientifique de Tadeusz Ważewski, l'auteur de plus de 100 mémoires, que les problèmes dont il s'occupait appartenaient aux plus actuels.

Depuis 1960 Ważewski a publié toute une série de travaux [100]-[106]. [108]-[112] sur les systèmes de commande. Ce sont les équations différentielles de la forme

$$
d x / d t=f(t, x, u),
$$

où $u$ est le vecteur de commande. L'équation (3) devient l'équation différentielle ordinaire, si l'on remplace le vecteur $u$ par une fonction $u(t)$, dite fonction de commande. Il on résulte que le problème initial de Cauchy pour l'équation (3) admet plusieurs solutions et qu'en général il y en a autant que des fonctions de commande admissibles. Les systèmes de commande interviennent dans les problèmes de commande optimale, dans lesquels il s'agit de trouver dans la classe de fonctions de commande admissibles celle qui est optinale au point de vue d'un critère donné d'avance.

Ważewski a fait la remarque suivante qui s'est montrée ensuite très féconde. Si le point $(t, x)$ étant fixé on désigne par $F(t, x)$ l'ensemble qui est l'image du domaine du paramètre de commande $u$, par l'intermédiaire de la transformation

$$
y=f(t, x, u),
$$


alors chaque solution du systène (3) est une solution du problène suivant: trouver une fonction absolument continue $x(t)$ satisfaisant pour presque chaque $t$ d'un intervalle convenable à la condition

$$
d x / d t \in F(t, x(t))
$$

Cette condition s'appelle équation différentielle au second membre multivoque ou bien équation aux orienteurs. La notion de l'orienteur a été introduite par Ważewski. Grâce à cette observation Ważewski a relié la théorie des systèmes de commande avec celle des équations au contingent qui a été développée avant la guerre par S. K. Zaremba en Pologne et par A. Marchaud en France. Ceci a conduit aux maints résultats importants et intéressants obtenus par Ważewski lui-même et par ses élèves.

En parlant des succès scientifiques de Ważawski il faut mentionner que son intérêt pour la physique, datant du début de ses études, a trouvé l'expression dans les travaux sur la corde vibrante [75], [76], [79].

Ważewski savait non seulement saisir des problèmes essentiels, mais par une analyse profonde il réussissait à trouver la méthode propre de solution qui se montrait souvent simple d'une façon inattendue. Les notions et les méthodes introduites par lui étaient toujours extraordinairement fécondes et servaient de point de départ aux investigations et aux généralisations. A côté de la méthode de retracto susmentionnée et de la théorie des équations aux orienteur's on pourrait indiquer d'autres exemples. On doit souligner que Ważewski concentrait son attention aussi sur les questions didactiques et méthodiques, ainsi que sur l'élégance des démonstrations mathématiques. L'unification de la démonstration d'une soixantaine de cas de la règle de de l'Hôspital en est un exemple frappant [49], [52]. Ici il faut remarquer que la règle de de l'Hôspital et ses généralisations conduisirent Ważewski au théorème sur les accroissements finis pour une fonction de la variable réelle et aux valeurs dans un espace de Banach [56]. Ce théorème servit de point de départ à la théorie des inégalités différentielles dans les espaces de Banach et aux constructions ingénieuses des solutions des équations différentielles dans ces espaces $[71],[72],[114],[115]$.

La formation des jeunes mathématiciens fut, à côté de son activité scientifique personnelle, la passion de Tadeusz IVażewski. C'était déjà parmi les étudiants de la première année que Ważewski savait trouver sans défaut des chercheurs futurs. Ważewski était maître à trouver des problèmes simples et intéressants sur lesquels il attirait l'attention des étudiants en leur faisant entreprendre des investigations à eux-mêmes. Ses cours étaient extrêmement vifs et fascinaient l'auditoire. Des problèmes intéressants et actuels que posait Ważewski ainsi que son talent et son enthousiasme contagieux pour la mathématique attiraient les étudiants les plus doués. Ważewski n'ignorait aucun effort de ses élèves, analysait et estimait 
chaque bonne idée. Au cas de quelque défaut il indiquait d'autres possibilités de traiter et de résoudre le problème. Des idées simples et frappantes trouvaient son accueil vif, souvent enthousiaste. Cette réaction vive de Ważewski aux résultats même très simples fut un encouragement additionnel pour ses élèves.

Les séminaires de Ważewski se caractérisaient par une analyse profonde et perspicace des questions traitées et par le soin porté à élucider toutes les difficultés. Aux séminaires de Ważewski, si l'on présentait les résultats d'autres auteurs, on cherchait toujours à révéler les points essentiels et l'idée directrice des théorèmes, à trouver leur genèse. Si le conférencier n'a pas réussi à le faire lui-même, on le faisait avec l'assistance des participants du séminaire. De cette façon on arrivait souvent à une conception nouvelle et plus générale du problème, à trouver une nouvelle et plus naturelle voie pour sa résolution, à découvrir de nouveaux résultats. Les séminaires de WVażewski étaient une vraie école scientifique.

Dans ses cours universitaires de même que dans ses conférences scientifiques Waźewski tenait à présenter l'idée directrice de la démonstration, à mettre en évidence la genèse du problème ou de la définition introduite. Il indiquait non seulement les conséquences d'une définition mais faisait entrevoir aussi la situation où les conditions d'une définitions sont en défaut. Il faut non seulement - disait-il - montrer aux étudiants ce qui se passe, si une propriété a lieu, mais aussi ce qui arrive, si elle est en défaut. C'était cette façon de présenter leurs propres résultats et ceux d'autres auteurs, que Ważewski demandait à ses élèves. Le fait que le résultat obtenu était correct ne le satisfaisait jamais. Il exigeait une démonstration aussi simple que possible, la mise en évidence de l'idée directrice et l'indication des hypothèses essentielles à l'aide des contre-exemples convenablement construits. Ważewshi attachait une grande importance à la façon dont on rédigeait les notes et il l'enseignait aussi à ses élèves. Il préparait ses élèves au travail scientifique de même qu'au travail pédagogique. Il veillait sur les premiers exercices et cours menés par ses élèves en leur donnant des conseils méritoriques et didactiques. Ważewski était maître infatigable. Il consacrait beaucoup de temps et de sentiment au travail avec les élèves. Il se souciait de leurs conditions matérielles, leur procurait des bourses en pays et à l'étranger, les présentait aux prix. Il était un patron bien soigneux de ses élèves. Il leur facilitait l'entrée dans le monde mathématique. Leur participation aux congrès et aux conférences l'engageait personellement, leurs succès lui faisaient plaisir.

La création do l'école des équations différentielles de Cracovie fut le couronnement de l'activité scientifique et pédagogique de Tadeusz Ważewski, maître et savant. C'est un succès sans pareil dans l'histoire de la mathématique polonaise après 1945. L'influence de cette école sur le développement des recherches dans certaines branches de la théorie 
qualitative des équations différentielles ordinaires est bien prononcée et distincte.

Plusieurs professeurs, dont trois sont déjà membres de l'Académie Polonaise des Sciences, sont sortis de l'école de Ważewski. Les élèves de Ważewski font une grande partie du centre mathématique de Cracovie.

L'école des équations différentielles de Cracovie s'est formée dans les années où le pays se levait des ruines de la guerre, où la methématique polonaise était ravagée par l'occupation fasciste. Ważewski a contribué à réconstruire la mathématique polonaise, à lui faire récuperer sa haute position dans le monde.

Ważewski entretenait des contacts scientifiques avec l'étranger en prenant part aux conférences et congrès internationaux et en faisant des cours sur l'invitation des universités étrangères. En 1959 il a visité par exemple les universités de Florence, Rome, Pise, Gênes, Lille et de Rennes ainsi que le Collège de France à Paris. Des visiteurs étrangers venaient pour faire connaissance de l'école de Ważewski.

Pour bien estimer les grands mérites de Ważewski il faut reculer à la période entre les deux guerres mondiales, quand a commencé le développement magnifique, durant jusqu'aujourdhui, des deux écoles mathématiques polonaise: école de topologie et de la théorie des ensembles à Varsovie et école de l'analyse fonctionnelle à Léopol. A cette époquo quelques mathématiciens seulement s'occupaient en Pologne des équations différentielles. Parmi eux il y avait pourtant des mathématiciens de grande renommée comme Stanisław Zaremba, Juliusz Schauder et Alfred Rosenblatt, mais qui travaillaient en isolation. Il n'était donc guère facile de concentrer l'attention des jeunes mathématiciens sur des problèmes de la théorie des équations différentielles, problèmes souvent très difficiles, et de former un groupe qui s'en occuperait. Cette oeuvre est due à Tadeusz Ważewski qui savait exiger et inspirer. C'est grâce à son initiative et grâce à l'Institut Mathématique de l'Académie Polonaise des Sciences que les recherches dans le domaine des équations différentielles commencèrent à se développer avec grand élan après 1945 en Pologne.

Bientôt après la guerre Ważewski fut élu membre correspondant de l'Académie Polonaise des Sciences et des Lettres. Dès le moment de la création de l'Académie Polonaise des Sciences il en fut nommé membre correspondant pour en devenir membre réel en 1957. En 1959 il fut élu président de la Société Polonaise de Mathématique, qui lui a conféré en 1967 le titre du membre honoraire. La même année il a reçu à l'Université Jagellone le titre de docteur honoris causa. Il était rédacteur $\mathbf{d u}$ journal Annales Polonici Mathematici. Deux fois le Prix Scientifique de l'Etat lui a été accordé. Pour ses mérites le Conseil d'Etat lui a conféré de hautes distinctions: la Croix de Chevalier et la Croix d'Officier do l'Ordre Polonia Restituta et deux fois l'Etandard du Travail de première 
classse. En 1949 il a reçu le Prix Scientifique de la Région de Cracovie et ensuite la Médaille d'Or de la Ville de Cracovie, pour laquelle il avait tant des mérites.

Ważewski était homme de grandes qualités de caractère. Sensible et timide, il était en même temps dur et déterminé à remplir les devoirs les plus difficiles dont il s'était chargé. Il savait choisir la vérité qu'on pouvait dire et celle qu'il fallait dire. La bonté s'alliait dans sa nature avec la sagesse. Il demandait peu pour lui en donnant avec générosité aux autres. Dans son allure il était prodigieusement charmant. Il était aussi spirituel, son esprit prenant næissance dans la précision de sa pensée critique. Très sensible aux grandes oeuvres de la science et de l'art i] a concentré son énérgie et sa perspicacité sur la mathématique qui fut la passion de sa vie.

Il restera toujours dans les coeurs de ceux qui ont eu la chance de collaborer avec lui et de puiser de sa sagesse et de sa bonté sans limite.

\section{Liste des publication de Tadeusz Ważewski}

[1] Sur un continu singulier, Fund. Mat. 4 (1923), p. 214-245.

[2] Sur les ensembles mesurables, C.R. Paris (1923), p. 69.

[3] Sur les courbes de Jordan ne renfermant aueune courbe simple de Jordan, Ann. Soc. Polon. Math. 2 (1923), p. 49-170.

[4] Kontinua prostowalne wowiazku z funkcjami i odwzorowaniami absolutnie ciaglymi, Dodatek do Roczll. Pol. Tow. Mat. (1927), p. 9-49.

[5] Pewne twierdzenie o funkcjach majqcych pochodnq. Wnioski, Księga Pamiątkowa Pierwszego Polskiego Zjazdu Mat. (1927), p. 115.

[6] Un théorème sur les fonctions dérivables, Ann. Soc. Polon. Math. 6 (1927), p. 83-92.

[7] Contributions à la théorie de la longueur, Ann. Soc. Polon. Math. 7 (1928), p. 1-38.

[8] Sur les jacobiens généralisés, Comptes Rendus du premier Congrès des Mathématiciens des Pays Slaves (1929), p. 210-213.

[9] Sur quelques propriétés des ensembles rectifiables, Comptes Rendus du premier Congrès des Mathématiciens des Pays Slaves (1929), p. 327.

[10] Un théorème sur le changement de variables dans les intégrales simples, Bull. Acad. Polon. Sci. Sér. A (1929), p. 203-2Il.

[11] Sur quelques points de la théorie de la longueur, Bull. Acad. Polon. Sci. Sér. A (1929), p. 11-16.

[12] Sur les jacobiens asymptotiques et le changement de variables dans les intégrales multiples, Bull. Acad. Polon. Sci. Sér. A (1930), p. 249-299.

[13] Zur Theorie des Unitätsproblems für Systeme von gewöhnlichen Differentialglei. chungen, Math. Z. 35 (1932), p. 553-562.

[14] Remarque sur un théorème de M. Bielecki, Ann. Soc. Polon. Math. 10 (1932), p. 42-44.

[15] Sur la stabilité des intégrales d'un système d'équations différentielles, C. R. Paris (1932), p. 1786.

[16] Sur un problème relatif à l'équation $\partial z / \partial x+Q(x, y) \partial z / \partial y=0$, Mathematica 8 (1933), p. $103-116$. 
[17] Sur l'unicité et la limitation des intégrales des équations aux dérivées partielles du premier ordre, Rend. della R. Acc. dei Lincei (1933), p. 372-376.

[18] Sur le domaine d'existence des intégrales de l'équation aux dérivées partielles du premier ordre linéaire, Ann. Soc. Polon. Math. 12 (1933), p. 6-15.

[19] Eine Verallgemeinerung des Montelschen Satzes über das Maximal und Minimalintegral auf Systeme von gewöhnlichen Differentialgleichungen, Aun. Soc. Polon. Math. 12 (1933), p. 72-80.

[20] Warunek jednotliwości calek ukladu równań różniczkowych zwyczajnyeh, Pamiętnik XIV Zjazdu Lekarzy i Przyrodników w Poznaniu, 11-15 IX. 1933, T. 1, p. 191-201.

[21] O zasięgu calek równań czqstkowych rzędu pierwszego, Pamiętnik XIV Zjazdu Lekarzy i Przyrodników w Poznaniu, 11-15 IX. 1933, T. 1, p. 187-199.

[22] Sur les équations aux dérivées partielles du premier ordre essentiellement non linéaires, Comptes Rendus du deuxième Congrès de Mathématiciens des Pays Slaves, Praha (1934), p. 171-172.

[23] Sur le domaine d'existence des intégrales de l'équation aux dérivées partielles du premier ordre, Ann. Soc. Polon. Math. 13 (1934), p. 1-9.

[24] Sur l'équation aux dérivées partielles du premier ordre essentiellement non linéaire, Ann. Soc. P'olon. Math. 13 (1934), p. 10-12.

[25] Sur les intégrales stables non périodiques des systèmes d'équations différentielles, Ann. Soc. Polon. Math. 13 (1934), p. 50-52.

[26] Sur les intégrales premières de l'équation $P(x, y) d x+Q(x, y) d y=0$, Mathematica 9 (1934), p. 179-181.

[27] Sur le domaine d'existence des intégrales d'une équation aux dérivées partielles du premier orlre, Bull. Acad. Polon. Sci. et Lettres, Sciences Math. 59 (1935), p. 1-4.

[28] Sur une classe de domaines, Prace Mat. Fiz. 44 (1935), p. 133-135.

[29] Sur les matrices dont les éléments sont des fonctions continues, Compositio Mathematica 2 (1935), p. 63-68.

[30] Sur l'appréciation du domaine d'existence des intégrales de l'équation aux dérivées partielles du premier ordre, Ann. Soc. Polon. Math. 14 (1935), p. 149-175.

[31] (avec S. K. Zaremha), Sur les ensembles de condensation des caractéristiques d'un système d'équations différentielles ordinaires, Ann. Soc. Polon. Math. 15 (1936), p. 24-33.

[32] Sur l'unicité et la limitation des intégrales de certains systèmes d'équations aux dérivées partielles du premier ordre, Ann. Mat. Pura Appl. Ser. IV, 15 (1937), p. $155-158$.

[33] Sur le problème de Cauchy relatif à un système d'équations aux dérivées partielles, Ann. Soc. Polon. Math. 15 (1936), p. 101-127.

[34] Quelques propriétés de caractère intégral de l'équation $P(x, y) d z / d x+Q(x, y) d z / d y$ $=0$, Comptes Rendus du Congrès International des Mathématiciens, Oslo T. II (1936), p. 49-50.

[35] Sur l'appréciation des intégrales des systèmes d'équations différentielles ordinaires et de leur domaine d'existence dans le cas des variables complexes, Ann. Soc. Polon. Math. 16 (1937), p. 97-111.

[36] Sur les intégrales premières des équations différentielles ordinaires, Ann. Soc. Polon. Math. 16 (1937), p. 145-161.

[37] Über die Bedingungen der Existenz der Integrale partieller Differentialgleichungen erster Ordnung, Matl. Z. 43 (1938), p. 522-532.

[38] Sur la méthode des approximations successives, Ann. Soc. Polon. Math. 16 (1938), p. 214-215. 
[39] Théorie des multiplicités régulières d'éléments de contact unis. Application aux transformations canoniques, Ann. Soc. Polon. Math. 17 (1945), p. 55-112.

[40] Sur l'évaluation du domaine d'existence des fonctions implicites réelles ou comple$x e s$, Ann. Soc. Polon. Math. 20 (1947), p. 81-120.

[41] (avec J. Szarski), Sur l'unicité des intégrales de l'équation de Clairaut modifiée, Ann. Soc. Polon. Math. 20 (1947), p. 157-160.

[42] (avec J. Szarski), Sur la relation entre le module d'un déterminant complexe et son déterminant réel associée, Ann. Soc. Polon. Math. 20 (1947), p. 1-6.

[43] Sur la limitation des intégrales des systèmes d'équations différentielles, Studia Math. 10 (1948), p. 48-59.

[44] Sur une méthode topologique de l'examen du phénomène asymptotique relativement aux équations différentielles ordinaires, Rendiconti della Accad. Nazionale dei Lincei, Sér. VIII, 3 (1947), p. 210-215.

[45] (avec J. Szarski) Sur un probleme roentgenographique de M. S. Majerek, Ann. Soc. Polon. Math. 20 (1974), p. 389-390.

[46] O matematyce $i$ jej zwiazku $z$ naukami innymi, Article dans le livre: Źycie, Czlowiek, Katowice (1974), p. 11-37.

[47] Sur les intégrales asymptotiques des équations différontielles ordinaires, Sprawozd. Tow. Nauk. Warsz. (22: III. 1947), p. 38-40.

[48] Sur un principe topologique de l'examen de l'allure asymptotique des intégrales des équations différentielles ordinaires, Ann. Soc. Polon. Math. 20 (1947), p. 279-313.

[49] Quelques démonstrations uniformes pour tous les cas du théorème de l'Hôspital. Généralisations, Prace Mat. Fiz. 47 (1949), p. 117-128.

[50] Exemples des groupes de transformations d'une droite en elle même qui dépendent de quatre paramètres essentiels, Prace Mat. Fiz. 47 (1949), p. 105-116.

[51] Sur les intégrales d'un système d'équations différentielles tangentes aux hyperplans caractéristiques issues du point singulier, Ann. Soc. Polon. Math. 21 (1949), p. 277-297.

[52] Une démonstration uniforme du théorème généralisé de l'Bôspital, Ann. Soc. Polon. Mat. 22 (1950), p. 161-169.

[53] Sur l'allure asymptotique des intégrales d'une équation différentielle non linéaire, Bull. Acad. Polon. Sci. et des Lettres, Sér. A, Sci. Math. (1949), p. 62-66.

[54] Sur certains lemmes relatifs au prolongement des intégrales des équations différentielles ordinaires, Bull. Acad. Polon. Sci. et des Lettres, Sér. A, Sci. Math. (1949), p. 73-74.

[55] Sur la coincidence asymptotique des intégrales de deux système d'équations différentielles, Bull. Acad. Polon. Sci. et Lettres, Sér. A, Sci. Math. (1949), p. 147-150.

[56] Une généralisation des théorèmes sur les accroissements finis au cas des espaces abstraits. Applications, Bull. Acad. Polon. Sci. et des Lettres, Sér. A, Sci. Math. (1949), p. 183-185.

[57] Systèmes des équations et des inégalités différentielles ordinaires aux deuxièmes membres monotones et leurs applications, Ann. Soc. Polon. Math. 23 (1950), p. $112-166$.

[58] Sur l'évaluation du domaine d'existence des fonctions implicites dans le cas des espaces abstraits, Fund. Math. 37 (1950), p. 5-24.

[59] Pewien sposób wyprowadzania wzorów na pochodna agregatu, iloczynu i ilorazu dwóch funkcji, Matematyka 3 (5) (1949), p. 30-35, et 4 (6) (1949), p. 35-38.

[60] $O$ dwu znaczeniach zapisu $x=c$, Matematyka 5 (12) (1950), p. 1-5.

[61] Elementy rachunku różniczkowego i calkowego, Kraków (Bratnia Pomoc Studentów AH) (1948), p. 1-197.

[62] Krótkie dowody pewnych lematów elementarnych z zakresu analizy, Sprawozdanie 
ze wspólnego 7 Zjazdu Polskich Matematyków i 3 Zjazdu Czechosłowackich Matematyków (communiqué), Praha (1949), p. 23.

[63] Une définition intuitive de la translation paralelle au sens de $M$. Levi-Civita, Sprawozdanie ze wspólnego 7 Zjazdu Polskich Matematyków i 3 Zjazdu Czechosłowackich Matematyków (communiqué), Praha (1949), p. 272.

[64] Sur les systèmes de deux équations différentielles linéaires dont les intégrales tendent asymptotiquement vers une ellipse, C. R. Soc. Sci. Lettr. do Varsovie, Cl. III, 51 (1948), p. 9-12.

[65] Remarques relatives aux certains théorèmes de M. Banachiewicz (I), Ann. Soc. Polon. Math. 22 (1949), p. 286-287.

[66] Théorème de M. Banachiewicz relatif à la méthode des moindres carrés. Remarques méthodologiques (II), Ann. Soc. Polon. Math. 22 (1949), p. 288.

[67] Twórczość i zaslugi naukowe Stanislawa Zaremby, Dodatek do Rocznika Pol. Tow. Mat. 21, Sprawozdanie z V Zjazdu Matematyków Polskich w Krakowie w dniach 29-30 maja 1947, Kraków (1951), p. 8-10.

[68] Sur certaines conditions de coincidence asymptotique des intégrales des systèmes d'équations différentielles, C. R. Soc. Sci. et Lettr. de Varsovie, Séance du 16. XII. 1949 (1950), p. $188-203$.

[69] Certaines propositions de caractère "épidermique" relatives aux inégalités différentielles, Ann. Soc. Polon. Math. 24 (1952), p. 1-12.

[70] Une condition nécessaire et suffisante pour qu'une fonction continue soit monotone, Ann. Soc. Polon. Math. 24 (1952), p. 111-119.

[71] Une modification du théorème de l'Hôspital liée au problème du prolongement des intégrales des équations différentielles, Ann. Polon. Math. 1 (1953), p. $1-12$.

[72] Une généralisation des théorèmes sur les accroissements finis au cas des espaces de Banach et application à la généralisation du théorème de l'Hôspital, Ann. Soc. Polon. Math. 24 (1953), p. 132-147.

[73] Sur les intégrales de branchement des systèmes des équations différentielles ordi. naires, Ann. Polon. Math. 1 (1953), p. 338-345.

[74] Sur l'évaluation du nombre des paramètres essentiels dont dépend la famille des intégrales d'un système d'équations différentielles ayant une propriété asymptotique, Bull. Acad. Polon. Sci. 1 (1953), p. 3-5.

[75] Sur une relation entre la façon de la mise en équation du problème physique avec la notion des solutions généralisées des équations aux dérivées partielles du second ordre, Bull. Acad. Polon. Sci. 1 (1953), p. 79-82.

[76] (avec J. Szarski) Sur une méthode de comparaison des équalions aux dérivées partielles du second ordre avec les équations différentielles ordinaires, Bull. Acad. Polon. Sci. 1 (1953), p. 6-10.

[77] Sur l'algorithmisation des méthodes des éliminations successives. Réponse à un article polémique de M. Th. Banachiewicz, Ann. Soc. Polon. Math. 24 (1953), p. 132-147.

[78] Sur certaines inégalites aux dérivées partielles relatives aux fonctions possédant la différentielle approximative, Ann. Polon. Math. 2 (1955), p. 219-233.

[79] (avec J. Szarski), Uwagi o rownaniu struny drgajacej, Zeszyty Naukowe Uniw. Jagiell. (1955), p. 5-14.

[80] Une méthode topologique de l'examen de l'allure asymptotique des intégrales des équations différentielles, Proceedings of the International Congress of Mathematics (1954) 3 (1956), p. 132-139.

[81] (avec K. Borsuk, K. Kuratowski, F. Leja, M. Marczewski, S. Mazur, J. Mikusiński, W. Orlicz, R. Sikorski et W. Slebodziński), Wplyw 
nowych metod matematycznych na rozwój klasycznych dyscyplin matematyki, Prace Mat. 2 (1956), p. 1-26. Traduction allemande dans la publication: Die Hauptreferate des 8. Polnischen Mathematikerkongresses vom 6. bis 12. September 1953 in Warschau, Deutscher Verl. der Wissenschaften in Berlin (1956), p. 45-68.

[82] Sur la structure de l'ensemble engendré par les intégrales non asymptotiques des équations différentielles, Bull. Acad. Polon. Sci. 3 (1955), p. 143-148.

[83] Sur la méthode de $A$. Pliś de déterminer le domaine d'existence de la solution du problème de Cauchy pour les équations aux dérivées partielles du premier ordre, Bull. Acad. Polon. Sci. 4 (1956), p. 131-135.

[84] Remarque sur un système d'inégalités intégrales, Ann. Polon. Math. 3 (1957), p. $210-219$.

[85] Une remarque sur l'allure asymptotique des intégrales des équations différentielles, Conférence au Congrès à Moscou en 1956.

[86] Quelques observations simples sur l'allure des intégrales des équations différen. tielles, Comptes Rendus du Congrès à Bucarest (1956).

[87] (avec C. Olech et $\mathrm{Z}$. Opial), Sur le problème d'oscillation des intégrales de l'équation $y^{\prime \prime}-g(x) y=0$, Bull. Acad. Polon. Sci. 5 (1957), p. 621-626.

[88] (avec A. Bielecki et J. Szarski), Równania różniczkowe oraz równania calkowe i rachunek wariacyjny, Bibliografia Matematyki Polskiej w X-leciu 1944-1954, Wiadom. Mat. 2 (1957), p. 61-73.

[89] (avec J. Szarski et $\mathrm{Z}$. Szm $\mathrm{z} \mathrm{dt}$ ), Une remarque sur la régularité des intégrales des équations différentielles hyperboliques du second ordre, Ann. Polon. Math. 6 (1959), p. 241-245.

[90] (avec A. Pliś), A uniqueness condition with a standard differential equation without uniqueness property, Bull. Acad. Polon. Sci. 6 (1958), p. 145-148.

[91] (avec J. Szarski) Sur l'existence des intégrales asymptotiques des équations différentielles issues d'un ensemble de dimension zéro, Colloq. Math. 6 (1958), p. $215-218$.

[92] (avec J. Szarski) Interprétation géométrique des conditions d'intégrabilité d'un système d'équations aux différentielles totales, Ann. Polon. Math. 6 (1959), p. 301-304.

[93] (avec Z. Mikolajska), Sur les opérations transformant séries convergentes en séries convergentes, Bull. Acad. Polon. Sci. 6 (1958), p. 615-618.

[94] Sur l'allure asymptotique des intégrales des équations différentielles ordinaires, Intern. Congr. of Math. 1958, Abstract of short communications, p. 69-70.

[95] Sur un problème asymptotique relatif au système de deux équations différentielles ordinaires, Annali di Matematica 49 (1960), p. $139-146$.

[96] Rola Instytutu Matematycznego $w$ dzialalności poszczególnych ośrodków matematycznych w Polsce, Wiadom. Mat. 3 (1960), p. 217-221.

[97] Sur une extension du procédé de $I$. Jungerman pour établir la convergence des approximations successives au cas des équations différentielles ordinaires, Bull. Acad. Polon. Sci. 8 (1960), p. 43-46.

[98] Sur un procédé de prouver la convergence des approximations successives sans utilisation des séries de comparaison, Bull. Acad. Polon. Sci. 8 (1960), p. 48-52.

[99] Sur la dérivabilité de la limite d'une suite des fonctions possédant une dérivée approximative unilatérale (cas de l'espace de Banach), Bull. Acad. Polon. Sci. 8 (1960), p. 301-305.

[100] Systèmes de commande et équations au contingent, Bull. Acad. Polon. Sci. 9 (1961), p. 151-155.

[101] O problemie optymalnego sterowania w przypadku nieliniowym, Archiwum Automatyki i Telemechaniki 7 (1962), p. 19-32. 
[102] Sur une condition d'existence des fonctions implicites mesurables, Bull. Acad. Polon. Sci. 9 (1961), p. 861-866.

[103] Sur une condition équivalente à l'équation au contingent, Bull. Acad. Polon. Sci. $9(1961)$, p. 865-867.

[104] Sur la sémicontinuité inférieure du ,tendeur" d'un ensemble compact variant d'une façon continue, Bull. Acad. Polon. Sci. 9 (1961), p. 869-872.

[105] Sur une généralisation de la notion des solutions d'une équation au contingent, Bull. Acad. Polon. Sci. 10 (1962), p. 11-15.

[106] Sur les systèmes de commande non linéaires dont le contredomaine de commande n'est pas forcément convexe, Bull. Acad. Polon. Sci. 10 (1962), p. 17-21.

[107] (avec A. Pliś), Functions with all partial derivatives arbitrarily prescribed at a point, Ann. Polon. Math. 12 (1962), p. 155-157.

[108] Sur quelques définitions équivalentes des quasitrajectoires des systèmes de commande, Bull. Acad. Polon. Sci. 10 (1962), p. 469-471.

[109] Équations au contingent et systèmes de commande, Intern. Congress of Mathematics 1962, Abstracts of short communications (1962), p. 204.

[110] On certain conditions of existence of periodic trajectories and quasitrajectories of the control system of ordinary differential equations, Non-linear vibration problems, Warszawa (1964), p. 376-377.

[111] Sur un système de commande dont les trajectoires coincident avec les quasitrajectoires du système donné, Bull. Acad. Polon. Sci. 11 (1963), p. 101-104.

[112] On an optimal control problem (in connection with the theory of orientorfields of A. Marchaud and S.K. Zaremba), Differential equations and their applications, Proceedings of the Conference held in Prague in September 1962 (Praha 1963), p. $229-242$.

[113] (avec J. Szarski), Stanislaw Zaremba, Studia z dziejów Katedr Matematyki, Fizyki i Chemii Uniwersytetu Jagiellońskiego (1964), p. 103-117.

[114] Sur la convergence des approximations successives pour les équations différentielles ordinaires au cas de l'espace de Banach, Ann. Polon. Math. 16 (1965), p. 231-235.

[115] Une méthode de construction des solutions approchées des équations différentielles ordinaires au cas de l'espace de Banach, Bull. Acad. Polon. Sci. 14 (1966), p. 133-136.

[116] Remarque sur un cas particulier de la méthode de Runge et Kutta, Funkcjalaj Ekvacioj 9 (1967), p. 287-290.

[117] On differential equations asymptotically induced by a sequence of discrete functions, Bull. Acad. Polon. Sci. 15 (1967), p. 551-555.

[118] Sur un système des inégalités intégrales ordinaires non linéaires, Bull. Acad. Polon. Sci. 17 (1969), p. 225-231.

[119] Arzela-like theorem with applications to differential equations and control theory, Differential equations and their applications, Proc. of the conf. held in Bratislava in September 1966, Bratislava 1969. 
